\title{
Therapeutics
}

\section{Vitamin and fatty acid supplements may reduce antisocial behaviour in incarcerated young adults}

Gesch B, Hammond S, Hampson S et al. Influence of supplementary vitamins, minerals and essential fatty acids on the antisocial behaviour of young adult prisoners. Brit J Psychiatry 2002 July; 181:22-8.

QUESTION: Do vitamin, mineral and fatty acid supplements reduce antisocial behaviour among young adults in prison?

\section{Design}

Randomised controlled trial. Clinicians, participants and outcome assessors were blind to treatment allocation.

\section{Setting}

Information from authors: One maximum security correctional institution in Aylesbury, United Kingdom. Recruitment began September 1996.

\section{Participants}

Information from authors: Participants were 231 prisoners, aged 18-21 years, typically serving long sentences for serious offences.

\section{Intervention}

Participants received dietary supplementation or placebo (after stratifying for prison wing). The supplementation group received a vitamin and mineral supplement based on $100 \%$ of the Reference Nutrient Intakes and essential fatty acid supplements in 4 daily capsules (1260 mg linoleic acid; $80 \mathrm{mg}$ gamma linolenic acid; $80 \mathrm{mg}$ eicosapentaenoic acid, and $44 \mathrm{mg}$ docasahexaenoic acid). The average time spent on supplementation or placebo was
142 days. There was $100 \%$ follow up for the intention to treat analysis.

\section{Main outcome measures}

Antisocial behaviour was measured using incidents adjudicated by Governor reports (serious incidents such as violence) and minor action reports (for instance, failure to comply with requirements) which had been 'proven by adjudication.'

\section{Main results}

Participants receiving supplements were $26.3 \%$ (95\% CI $8.3 \%$ to $44 \%$ ) less likely to be reported for antisocial behaviour than those who received placebo (mean difference 11.8 less infringements in the supplement group, $p=0.03$ ). No participant withdrew due to ill effects of supplementation and there were no adverse events reported.

\section{Conclusions}

Vitamin, mineral and fatty acid supplements reduced antisocial behaviour, including violence, in young adult prisoners.
Source of funding

Natural Justice

Charity. Nutritional

supplements supplied

by Pharmaceuticals Ltd

and Unigreg Ltd.

For correspondence:

CB Gesch, University

Laboratory of

Physiology, University

of Oxford, UK.

bernard.gesch@

physiol.ox.ac.uk

\section{COMMENTARY}

Well designed studies that consider the aetiology of anti-social behaviour are rare. Gesch $e t$ al should therefore be congratulated for their valuable contribution. In California, Schoenthaler has considered the impact of micro-nutrient supplementation for many years. ${ }^{1}$ The ability of a group in another country to replicate his findings increases our confidence in these observations. There are no details about participants' ages, however, let alone whether their history was one of violence or petty crime. It would have been helpful to provide some detail about the criminal history of the sample.

It is probable that the findings have more practical implications in prisons than elsewhere, although some school-based studies suggest a similar effect. It may be more difficult to examine the benefits of supplementation in a non-institutionalised population. Few would deny the role of social interaction and subcultural norms in generating anti-social behaviour. Isolating a group from their normal social environment and placing them in a uniform and highly regimented social setting maximises the potential impact of biology. In addition, in a setting where half of the inhabitants are also consuming supplements, the likelihood of social interaction escalating into violence may be further decreased.

If the findings can be generalised to other prison populations, regular consumption of supplements should be considered. In principle, people should obtain micro-nutrients from an adequate diet, but it is difficult to define 'sufficient' quantities. In this instance, the youths' diet was already good. The participants' diet may have been of poorer quality outside prison. If so, dietary factors might prove to be more significant when nutrition is even more compromised. It would have been interesting to relate changes in behaviour after supplementation to the quality of diet consumed. Did those with a poorer diet benefit to a greater extent?

Gesch et al incorrectly suggest that "deficits in nutrition can be readily identified and remedied." Given the multitude of roles played by micro-nutrients, it would be difficult to establish specific mechanisms, especially given the wide range of nutrients consumed. There are marked differences in the need for micronutrients resulting from individual differences in the ability to absorb, transport and metabolise nutrients. Yet there is no reliable way of measuring the nutritional needs of an individual. Even biochemical assays fail to deal with individual differences in requirements. Findings in this area will prove difficult to implement unless large groups receive supplements or improved diets in an indiscriminate manner.

The first signs of subclinical dietary deficiency may be psychological in nature. ${ }^{2}$ Gesch $e t$ al add to a growing body of well controlled studies that suggest that micro-nutrient supplementation improves psychological functioning, including mood and intelligence. ${ }^{3-5}$ The findings add to a wider debate: the benefits of supplementation in those who are well fed. According to received wisdom, this group of youths should have been obtaining their daily requirements through food. The exception was selenium intake, although there is evidence that this lowers mood rather than violence. ${ }^{4}$ Recommended dietary intakes traditionally aimed to prevent deficiency and disease. Evidence that supplements are beneficial in those consuming the recommended intake may lead us to establish dietary norms for optimal behavioural functioning.

Professor David Benton cerated juveniles. J Nutr Environ Med 1997; 7: 343-52.

Benton D. Vitamin-mineral supplements and intelligence. Proc Nutr Soc 1992; 51: 295-62.

Benton D. Micro-nutrient supplementation and the intelligence of children. Neurosci Biobehav Rev 2001; 25: 297-309.

Benton D. Selenium intake, mood and other aspects of psychological functioning. Nutr Neursci 2002, 5:363-74.

Benton D, Griffiths R, Haller J. Thiamine supplementation mood and cognitive functioning. Psychopharmacol 1997; 129: 66-71.

Author's response

This study used a double-blind design to isolate the effect of broad-spectrum nutrients on behaviour. Prisoners did not reach the Dietary Reference Intakes for many nutrients due to poor food choices. Interaction between the active and placebo groups in the prison would reduce any observed effect in a between-groups comparison. In terms of the applicability of these findings, it is not where you eat that is important, but the nourishment it provides. These results may therefore be applicable outside prisons where poor diets are consumed. This is supported by a study in schools which found comparable effects. We have also completed a pilot of supplementation for offenders in the community and plan a large community-based trial. Direct measures of nutritional status from blood are required to associate changes in behaviour with nutritional status. This will be done when replicating this study, as will an examination of mediating mechanisms. 